\title{
APPLICATION OF HIGH-PERFORMANCE COUNTER CURRENT CHROMATOGRAPHY FOR SEPARATION OF COUMARINS FROM HERACLEUM MANTEGAZZIANUM
}

\section{(C) Skalicka-Woźniak K, Stępień P, Głowniak K}

Department of Pharmacognosy with Medicinal Plant Unit, Medical University in Lublin, 1 Chodzki, 20-093 Lublin, Poland, E-mail address: kskalicka@pharmacognosy.org

Coumarins are nowadays an important group of organic compounds from natural and synthetic sources that are useful in a number of fields. Because they are known for different pharmacological properties, their separation and purification is a very important task. A high-performance counter-current chromatography (HPCCC) method is an efficient preparative technique offering a series of advantages over conventional chromatography like elimination of irreversible adsorption of sample or easy scaling-up. HPCCC method was applied for the preparative separation and purification of major coumarins from the fruits of Heracleum leskowii.
A scale-up process from analytical to preparative in a very short time was developed. Different experiments with mixtures of $n$-hexane, ethyl acetate, methanol and water in reverse phase system were performed. Coumarins such as angelicin, bergapten, imperatorin and some not unidentified coumarin-type compounds were obtained. The components purified and collected were analyzed by high-performance liquid chromatography with UV-DAD.

Acknowledgments: This work was financially supported by Grant no N N405617538 from the Polish Ministry of Science and Higher Education.

\section{SUB-CHRONIC TOXICITY STUDY OF THE RAMBUTAN RIND EXTRACT IN MALE WISTAR RATS}

\section{(C) Thinkratok Aree ${ }^{1}$, Suwannaprapha Parin ${ }^{2}$, Srisawat Rungrudee ${ }^{1}$}

'School of Biology, Institute of Science, Suranaree University of Technology, 111 University Avenue, Suranaree district, Muang, Nakhon Ratchasima, Thailand

${ }^{2}$ Faculty of Veterinary Science, Mahidol University Salaya Campus, 999 Phutthamonthon Sai 4 Road, Salaya, Phutthamonthon, Nakhonpathom, Thailand

The fruit rind of rambutan (Nephelium lappaceum L.) has been used in traditional medicine for centuries and can be considered as an easily accessible source of natural antioxidant and antibacterial, anti-herpes simple virus type 1 , antiproliferative, and anti-hyperglycemic activities. However, toxicological data of this extract has not been reported. This study aimed to investigate sub-chronic toxicity of the rambutan rind extract (RRE) in male Wistar rats. Rats were daily orally administered of double deionized distilled water, 500, 1000 and $2000 \mathrm{mg} / \mathrm{kg}$ of the RRE at a dosing volume of $2 \mathrm{ml} / \mathrm{kg}$ for 30 days $(n=8$ each). All rats were daily observed for mortality, signs of toxicity, food consumption and weekly body weight. Relative organ weight (ROW) of heart, liver, kidneys, spleen and testes, plasma biochemical parameters [triglyceride, total cholesterol, glucose, creatinine, blood urea nitrogen (BUN), aspartate aminotransferase (AST) and alanine aminotransferase (ALT)] and hematological parameters [red blood cells, hemo- globin, hematocrit, platelet count, white blood cells, neutrophil, lymphocyte, monocyte, eosinophil and basophil] were examined at the end of the observation period. No mortality and toxicity sign was found after daily administration of the RRE up to $1000 \mathrm{mg} / \mathrm{kg}$. There was one mortality after administration of $2000 \mathrm{mg} / \mathrm{kg}$ RRE for 6 days. In this group, signs of toxicity (hypo-activity and lethargy) were observed. The RRE significantly decreased body weight gain, food consumption, plasma triglyceride and BUN levels, and lymphocyte. The RRE caused significant increases in ROW of testes and heart, platelet count, neutrophil and eosinophil. There was no liver toxicity since no change in AST, ALT or liver weights were found. Therefore, the no-observed-adverse-effect level (NOAEL) was $1000 \mathrm{mg} / \mathrm{kg}$ due to absence of toxic effect. The results obtained from this study will help for future clinical studies of the medicinal safety and in vivo experimental studies of the pharmacological potentialities of the RRE. 